\title{
A road to obstetric fistula in Malawi: capturing women's perspectives through a framework of three delays
}

This article was published in the following Dove Press journal: International Journal of Women's Health

\author{
Josephine Changole' \\ Viva Combs Thorsen' \\ Ursula Kafulafula ${ }^{2}$ \\ 'Department of Community Medicine \\ and Global Health, University of \\ Oslo, Oslo, Norway; ${ }^{2} \mathrm{Kamuzu}$ College \\ of Nursing, University of Malawi, \\ Blantyre, Malawi
}

Introduction: Obstetric fistula (OF) is a devastating birth injury, which leaves a woman with leaking urine and/or feces accompanied by bad smell, a situation that has been likened to death itself. The condition is caused by neglected obstructed labor. Many factors underlie fistula formation, most of which are preventable. The main purpose of this study was to explore labor and childbirth experiences of women who developed OF with a focus on accessibility of care in the central region of Malawi.

Methods: We conducted semi-structured interviews with 25 women with OF at Bwaila Fistula Care Center in Lilongwe and in its surrounding districts. We interviewed 20 women at Bwaila Fistula Care Center; additional five women were identified through snowball sampling and were interviewed in their homes. Data were categorized using Nvivo 11 and were analyzed using thematic analysis. The three delays model by Thaddeus and Maine was used for data analysis.

Findings: The majority of women in our study suffered from OF with their subsequent pregnancies. All women experienced delays in one form or another consistent with the three-phase delays described by Thaddeus and Maine. Most of the participants (16) experienced two delays and 15 experienced second-phase delay, which was always coupled with the other; nine participants experienced delay while at the hospital. None of the participants experienced all three delays. Most decisions to seek health care when labor was complicated were made by mothers-in-law and traditional birth attendants. All but two delivered stillborn babies.

Conclusion: Testimonies by women in our study suggest the complexity of the journey to developing fistula. Poverty, illiteracy, inaccessible health facilities, negligence, lack of male involvement in childbirth issues, and shortage of staff together conspire to fistula formation. To prevent new cases of OF in Malawi, the above mentioned issues need to be addressed, more importantly, increasing access to skilled attendance at birth and emergency obstetric care and promoting girls' education to increase their financial autonomy and decision-making power about their reproductive lives. Also men need to be educated and be involved in maternal and women's reproductive health issues to help them make informed decisions when their spouses end up with a complicated labor or delivery.

Keywords: childbirth experience, delays, obstetric fistula, Malawi

\section{Background}

Dhangole Department of Community Medicine and Global Health, University of Oslo, P.O. Box II 30 Blindern, N-03 I8

Oslo, Norway

Tel +4722850587

Email josephine.changole@

studmed.uio.no
Obstetric fistula (OF) is a devastating birth injury, which leaves a woman with leaking urine and/or feces accompanied by a bad smell, a situation that has been likened to death itself. ${ }^{1}$

The condition is primarily caused by a neglected, prolonged, obstructed labor. ${ }^{2} \mathrm{OF}$ is extremely rare in high-income countries due to sufficient and effective universal 
emergency obstetric care, ${ }^{3}$ rendering its preventability unquestionable. Unfortunately, such services are limited or unavailable in low- and middle-income countries, escalating the burden of fistula in these countries. It is estimated that 2 million girls and women in South Asia and Sub-Saharan Africa are currently living with OF. ${ }^{4}$ Many factors contribute to fistula formation in the affected regions. Apart from unavailable or inaccessible emergency obstetric care services that has been mentioned earlier, the following factors may also predispose a woman to obstructed labor and consequently OF: inadequately developed pelvises as in teenage pregnancies, being stunted from childhood malnutrition, having poor health, dependency on others for decisions and support for health, and also ascribing to harmful cultural practices that promote early marriages and home deliveries. An even more critical factor that has been shown to be significant in fistula formation and other maternal morbidities and mortalities is timing, more specifically delays in acting, be it at the individual level or facility level. In Kenya, ${ }^{5}$ researchers observed that women with OF in their study were more likely to have made a decision to seek delivery assistance 6 hours after the onset of labor, to have traveled $>2$ hours to reach a health facility, and to have labored for $>24$ hours. ${ }^{5}$ Similarly, researchers found that the majority (69\%) of women with fistula had experienced two or more delays in receiving care when labor was obstructed. ${ }^{6}$ Reasons for the delays in that study were long distances to the health facility, lack of money and transportation, fear of hospital costs, fear of negligence, and abusive behavior of some health workers.

Currently, only limited studies in Malawi have investigated personal childbirth experiences of women with OF, focusing on delay. Previous studies that focused on delays were on maternal deaths. ${ }^{7,8}$ Thorsen et $\mathrm{al}^{7}$ used the three delays model to identify the sociocultural and facility-based factors that contributed to maternal deaths in the district of Lilongwe, Malawi, and found that upon reaching the facility most women received late treatment due to delayed referrals, missed diagnoses, lack of essential drugs and supplies, and severe mismanagement. Similar findings were also reported in a study on factors associated with maternal deaths in the district of Mangochi in Malawi. ${ }^{8}$ Neither of these studies focused on maternal morbidity. Moreover, previous studies in Malawi on OF focused on the prevalence of fistula, ${ }^{9,10}$ experiences of living with fistula pre- and postsurgical repair, ${ }^{11-13}$ and the outcomes of surgical repair. ${ }^{14}$ In the present study, we sought to understand labor and delivery experiences of women who developed OF in Malawi, using the three delays model described below. The model was deemed appropriate because the circumstances that surround women who die from complications during pregnancy or childbirth are similar to those who suffer from acute or chronic morbidities, including fistula. Moreover, in the case of OF, we are able to glean insights from first-hand accounts from the women themselves of what transpired.

\section{Theoretical framework}

In this study, we used the three delays model by Thaddeus and Maine ${ }^{15}$ for data analysis. Thaddeus and Maine ${ }^{15}$ group health care-related delays into three interrelated phases as described below.

\section{First delay: delay in recognizing illness and deciding to seek care}

This is influenced by the type of decision makers involved, woman's autonomy, distance to health facility, previous experiences with the health care system, perceptions about the condition, and health knowledge, which might prevent or promote early recognition of danger signs and cultural and traditional beliefs.

\section{Second delay: delay in reaching a medical facility after the decision to seek care has been made}

This type of delay can be affected by distance to health care facilities, availability and cost of transportation, poor roads and infrastructure, geographical obstacles, and delayed referrals from one health care facility to another.

\section{Third delay: delay in receiving adequate care upon reaching the medical facility}

This type of delay includes factors that affect timely treatment once the woman reaches a health care facility such as shortages of supplies, equipment, and trained personnel; competence of available personnel; and quality of care.

\section{Methods \\ Study design}

We employed a qualitative research design, with a social constructivist perspective. ${ }^{16}$ Social constructivism proposes that realities are constructed through lived experiences and interaction with others and that "meanings are derived from perceptions, experiences, and actions in relation to social context."16 This perspective was deemed appropriate because we wanted to explore and understand labor and delivery experiences of women who developed fistula as expressed by the sufferers themselves without limiting their experiences by predetermined responses as the case with a positivist perspective. ${ }^{17}$ 


\section{Context and study setting}

Malawi is one of the poorest countries in Sub-Saharan Africa, with an estimated population of over 17 million. ${ }^{18}$ The country is divided into three regions, which are further subdivided into 28 administrative districts. Malawi has three levels of health service delivery: primary, secondary, and tertiary. Primary level facilities offer basic health services and are the entry points for the formal health care delivery system. It includes health centers, dispensaries, and health posts. Staff at this level includes nurse midwife technicians, enrolled nurse midwives, clinical officers (COs), and medical assistants. All these cadres, except for medical assistants, undergo a 3-year similar training program that prepares them to perform as skilled birth attendants (SBAs), to manage normal deliveries, and to refer complications such as obstructed labor; the primary cause of OF. District hospitals constitute the secondary level of health care and provide specialized services to patients referred from the primary health care level, through outpatient and inpatient services and community health services. These services are enhanced by the provision of adequate specialized supportive services, and they have the capacity to provide comprehensive emergency obstetric and neonatal care. Tertiary health care level consists of highly specialized services. At this level, central hospitals and other specialist hospitals provide care for specific disease conditions or specific groups of patients. These facilities are referral centers for the district hospitals and in some cases for rural hospitals, community hospitals, and health centers. These different levels are linked to one another through an elaborate referral system that has been established within the health system. Pregnant women first visit the primary-level facility and are referred to the next level in case of an obstetric complication. ${ }^{19}$ The common mode of transportation linking the facility levels is ambulance, whose availability and functionality determine how fast the individual - in this case, the pregnant woman - gets to the next level for appropriate care.

Our study was limited to the central region of Malawi. Central region has an estimated population of 7 million $^{20}$ and is subdivided into nine administrative districts. Each district consists of several health centers, which refer to a district hospital. The region is predominantly inhabited by Chewa tribe. Chewa people culturally practice matrilineal system of marriage and are virilocal. ${ }^{21}$ Due to the virilocal system, issues of pregnancy and childbirth are usually decided upon by mothers-in-law or sisters-in-law and uncles called "mwinimbumba," regardless of their level of knowledge about childbirth issues. Chewa people are an agriculturebased society, ${ }^{22}$ and farming is the primary income generator through growing maize and tobacco. This makes them more dependent on rains and, thus, vulnerable to poverty when rains are poor. The majority of people live in the lower wealth quintile compared to the other two regions in the country. The majority of women have no or some primary education; with just a few completing their primary and secondary education. According to Malawi Demographic Health Survey (MDHS) 2016, ${ }^{23}$ the majority of women $(97 \%)$ in the region had skilled antenatal care (ANC) in the 5 years prior to the survey, but only $90 \%$ had deliveries at a health facility; the rest were outside the health facility, assisted by relatives, neighbor, or traditional birth attendants (TBAs). Women also suffered more stillbirths compared to their counterparts in the south and north. This could be because they also had more challenges in accessing maternal health services compared to their counterparts. ${ }^{23}$

\section{Maternal health in Malawi}

Malawi failed to achieve the Millennium Development Goal number 5 in 2015, like most low-income countries in Sub-Saharan African region. However, maternal deaths have decreased since the year 2000 from 1,123 per 100,000 live births to 439 per 100,000 live births in $2016 .{ }^{23}$ Despite the decrease, the rate is still the highest in the Sub-Saharan region. The reduction reflects improved utilization of antenatal and delivery services. The decrease in home deliveries from $43 \%$ in 1992 to $7 \%$ in 2016 could be attributed to the ban on deliveries by TBAs in 2007. ${ }^{23}$

Currently, there is only one care center that treats OF, Bwaila Fistula Care Center (BFCC), which is situated in Lilongwe (central region). The center was established by the Freedom from Fistula Foundation in 2010. ${ }^{24}$ Before 2010, women were managed in tertiary hospitals located in Blantyre and Zomba in the southern region, Lilongwe in the central region, and Mzuzu in the northern region. However, due to shortage of surgeons with technical skills to manage fistula, many women continued to suffer from the condition for very long periods of time. ${ }^{25}$

Currently, it is estimated that 1.6 per 1,000 women of reproductive age (15-49 years) are living with fistula. ${ }^{9}$ Anecdotal reports state that 20,000 ( 5 per 1,000) women are living with this condition. The MDHS $2016^{23}$ reports that $1 \%$ of 24,562 women $(n=246)$ who participated in the survey had experienced OF between 2010 and 2015.

\section{Study participants}

Twenty-eight women diagnosed with OF participated in this study. To participate, the women had to be diagnosed with vesicovaginal fistula or rectovaginal fistula or both, admitted at BFCC, waiting for surgery or recovering from surgery, living within the Center's catchment area and within the 
central region, regardless of her age and duration of living with fistula, and willing to participate in the study. Three of the women refused to participate, making the final number of participants 25 . Twenty of the women were purposively selected from BFCC, while five were recruited through snowball sampling through those who had been recruited at BFCC. The women recruited through snowball sampling were visited in their homes and invited to participate in the study. Some women had previously been diagnosed with fistula, but had not yet sought treatment at BFCC.

\section{Data collection}

Semi-structured interviews, ${ }^{17}$ which lasted between 30 and 90 minutes, were conducted by the first author. The questions focused on demographic information and historical accounts of the pregnancy that led to fistula development. To increase trustworthiness, ${ }^{17}$ the first author conducted follow-up interviews with 10 participants in order to clarify, resolve, and confirm issues based on the responses from the participants' earlier interviews.

\section{Data analysis}

The interviews were analyzed concurrently with data collection process. All the interviews were transcribed verbatim and translated from Chichewa to English by the first author and three research assistants. Three transcripts were given to a linguistic teacher at a secondary school, with a Bachelor's degree in Education, to back translate to Chichewa to ensure that the meanings had not been lost in translation, and there were no significant differences. The first author did the final editing by checking all the transcripts against audio recordings to ensure accurate transcription and translation. A transcript was further checked against an audio recording whenever something did not make sense while reading it. The English transcripts were used for analysis, and they were crosschecked with the original Chichewa transcripts to ensure a correct interpretation throughout the process. The process was guided by a thematic analysis approach that was both deductive and inductive in nature. ${ }^{26}$ Deductively, previous studies and literature provided information on childbirth experiences of women who developed fistula in other countries. ${ }^{6}$ The three delays model helped determine into which category(-ies) each reported factor had been aligned. In other words, the data were reviewed/ analyzed with certain preconceived categories derived from the aforementioned theoretical model while allowing for themes to emerge directly from the data using inductive coding. Inductively, the transcripts were carefully read sentence by sentence to obtain a sense of the content as narrated by the participants and to identify the emerging themes.
Phrases and sentences related to labor and delivery experiences were coded in the margin of the transcript sheets, and codes with similar content were combined into categories. Nvivo 10 software was used for data management. Several rounds of discussions among the co-authors were necessary to strengthen the credibility and integrity of the findings. ${ }^{17,49}$ To ensure confirmability, all the co-authors reflected, discussed differences in the interpretation of data, and agreed on the categorization. ${ }^{17,27,49}$

\section{Ethical consideration}

The study was conducted in compliance with the principles of the Declaration of Helsinki. ${ }^{28}$ The study was approved by the College of Medicine Research Ethics Committee (COMREC) in Malawi; protocol number P.03/15/1711; but did not need an explicit permission from the obligation of the Act on medical and health research as stated by the Regional Ethics Committee (REK) in Norway; protocol number 2014/2040/REK, but was registered with the Norwegian Center for Research in Norway (NSD) ref: 43,620. All informants, including one mother under the age of 18 years, gave both oral and written informed consent as approved by the COMREC in Malawi. Participants gave consent for the responses from their interviews to be used in this manuscript. All transcripts were anonymized, and pseudonyms were used in this report to protect the participants' identities and to ensure confidentiality. Voluntary participation was emphasized. Women were given an equivalent of $\$ 2$ for transport to the hospital for those recruited through snowball sampling or back to their homes if recruited at the care center. This was done at the end of the interview to avoid undue inducement. ${ }^{28,49}$

\section{Strengths and limitations of the study}

The findings from this study add to the existing knowledge about OF by revisiting the three-delays theoretical framework from the perspective of the women who had survived the condition and can give firsthand accounts of what happened during labor and childbirth. The methods used are described in detail and may be transferable and adapted to settings and populations similar to those of our study. The first author is a nurse midwife, conversant with $\mathrm{OF}$, and she is a local researcher conversant with Malawian culture, which could affect the way data were collected and analyzed. To minimize researcher bias, she had an ongoing discussion with the co-authors during the interview guide development, data collection, data analysis, and manuscript development. Being a Malawian could have forced women to participate to help a fellow Malawian. To minimize this effect, voluntary 
participation was emphasized. Another limitation is that we could not separate iatrogenic fistula from those exclusively caused by obstructed labor, but whatever the case, most women had an operation after labor was already obstructed implying that the process of fistula formation could already have been underway, and also making cesarean delivery difficult which could have potentially led to iatrogenic fistulas. Some of the interviews were conducted at the health facility where the participants received fistula care, which might have influenced their responses; however, voluntary participation was emphasized, and participants were assured that their care will not be affected by their decision to participate or not. Another limitation is that our population was narrowly defined, and it excluded health care providers and TBAs who also play a role in fistula formation, making it difficult to confirm some of the participants' accounts.

\section{Findings}

\section{Characteristics of participants}

A total of 25 women participated in the study. Of those who knew, their age ranged from 16 to 67 years. The majority had no formal education. The majority attended ANC at a health care facility or at a TBA. Table 1 summarizes the characteristics of the participants.

\section{Experiences of delay}

Narratives from participants in our study suggested that they all experienced a delay in one way or another before they received obstetric care. Most of the participants (16) experienced two delays, 15 experienced second-phase delay (reaching a health care facility), which was always coupled with one other delay, and nine participants experienced delay once they arrived at the hospital (third-phase delay). None of the participants experienced all three delays. The onset of the first delay involved not seeking help immediately after they felt labor pains; they waited for labor to progress. Second, the decision to seek medical help when a complication occurred was also delayed. Due to distance and lack of transportation, they took an even longer time to get to the hospital, where they experienced the third and final delay in receiving appropriate care for their complication. The three delays are summarized in Table 2.

In the following sections, we present the experiences of these delays as narrated by the participants.

Table I Characteristics of participating women with fistula

\begin{tabular}{|c|c|c|c|c|c|c|c|c|c|c|}
\hline ID & $\begin{array}{l}\text { Age } \\
\text { group }\end{array}$ & $\begin{array}{l}\text { Years in } \\
\text { school } \\
\text { (range) }\end{array}$ & Parity & $\begin{array}{l}\text { Range of } \\
\text { children } \\
\text { alive }\end{array}$ & $\begin{array}{l}\text { Pregnancy } \\
\text { causing } \\
\text { fistula }\end{array}$ & $\begin{array}{l}\text { ANC at least } \\
\text { attended } \\
\text { once }\end{array}$ & $\begin{array}{l}\text { Type of } \\
\text { delivery }\end{array}$ & $\begin{array}{l}\text { \# of days } \\
\text { in labor }\end{array}$ & $\begin{array}{l}\text { Fetal } \\
\text { outcome }\end{array}$ & $\begin{array}{l}\text { Years/month } \\
\text { (m) with } \\
\text { fistula }\end{array}$ \\
\hline I & $33-42$ & $0-3$ & 3 & $2-3$ & 2 & Yes & CS & 3 & SB & 17 \\
\hline 2 & DK & $0-3$ & 7 & $4-5$ & 7 & Yes & CS & I & SB & 10 \\
\hline 3 & $16-32$ & $4-7$ & I & $0-1$ & I & Yes & CS & 5 & SB & I \\
\hline 4 & $16-32$ & $8-12$ & I & $0-1$ & I & Yes & CS & I & SB & $5 m$ \\
\hline 5 & $16-32$ & $4-7$ & 4 & $2-3$ & 4 & Yes & CS & I & SB & $4 m$ \\
\hline 6 & $16-32$ & $0-3$ & 6 & $2-3$ & I & Yes & CS & 2 & SB & 16 \\
\hline 7 & $43-67$ & $0-3$ & 6 & $2-3$ & 6 & Yes & CS & I & SB & 19 \\
\hline 8 & DK & $0-3$ & 6 & $4-5$ & 6 & No & CS & 2 & SB & 4 \\
\hline 9 & $33-42$ & $0-3$ & 2 & $0-1$ & 2 & No & CS & 2 & SB & 20 \\
\hline 10 & $33-42$ & $0-3$ & 2 & $0-1$ & 2 & Yes & CS & 0 & SB & 19 \\
\hline II & $43-67$ & $0-3$ & 2 & $0-1$ & 2 & No & CS & I & SB & 21 \\
\hline 12 & $43-67$ & $0-3$ & 3 & $0-1$ & 3 & No & CS & 1 & SB & 45 \\
\hline 13 & $43-67$ & $0-3$ & 2 & $0-1$ & 2 & Yes & CS & 2 & SB & 27 \\
\hline 14 & $16-32$ & $0-3$ & 5 & $2-3$ & 5 & Yes & CS & I & Alive & $3 m$ \\
\hline 15 & $16-32$ & $8-12$ & 2 & $2-3$ & 2 & Yes & Vaginal & I & Alive & I \\
\hline 16 & $16-32$ & $0-3$ & I & $0-1$ & I & Yes & Vaginal & I & SB & $4 m$ \\
\hline 17 & $33-42$ & $4-7$ & 2 & $0-1$ & I & Yes & Vaginal & 3 & SB & 15 \\
\hline 18 & $16-32$ & $8-12$ & 4 & $2-3$ & 4 & Yes & Vaginal & 2 & SB & Im \\
\hline 19 & $33-42$ & $0-3$ & 8 & $2-3$ & I & No & Vaginal & I & SB & 19 \\
\hline 20 & DK & $0-3$ & 7 & $2-3$ & 6 & No & Vaginal & 2 & SB & DK \\
\hline 21 & $33-42$ & $4-7$ & I & $0-1$ & I & No & Vaginal & 2 & SB & 12 \\
\hline 22 & DK & $0-3$ & 5 & $0-1$ & 5 & Yes & Vaginal & I & SB & 13 \\
\hline 23 & DK & $0-3$ & 13 & $0-1$ & I & Yes & Vaginal & I & SB & 47 \\
\hline 24 & $33-42$ & $4-7$ & 5 & $2-3$ & 3 & Yes & Vaginal & 2 & SB & 12 \\
\hline 25 & DK & $0-3$ & 5 & $0-1$ & 5 & Yes & VDO & I & SB & 15 \\
\hline
\end{tabular}

Note: Reproduced from Changole J, Thorsen VC, Kafulafula U. “I am a person but I am not a person": experiences of women living with obstetric fistula in the central region of Malawi. BMC Pregnancy Childbirth. 2017;17(I):433.49

Abbreviations: ANC, antenatal care; CS, cesarean section; DK, does not know/remember; VDO, vaginal destructive operations; SB, stillbirth. 


\begin{tabular}{|c|c|c|c|c|c|c|c|c|c|c|c|c|c|c|c|}
\hline 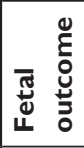 & 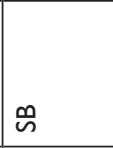 & $\ddot{n}$ & $\ddot{n}$ & $\ddot{n}$ & $\ddot{\omega}$ & $\ddot{n}$ & $\ddot{n}$ & $\ddot{n}$ & कि & $\ddot{n}$ & m & $\ddot{s}$ & $\ddot{n}$ & 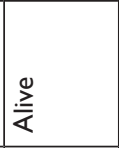 & 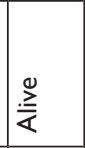 \\
\hline 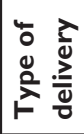 & $\tilde{u}$ & $\tilde{U}$ & $\breve{u}$ & $\tilde{U}$ & $\tilde{U}$ & $\tilde{u}$ & U & $\tilde{u}$ & $\breve{u}$ & $\tilde{U}$ & $\tilde{u}$ & $\tilde{u}$ & $\tilde{u}$ & $\tilde{u}$ & 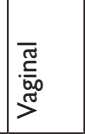 \\
\hline 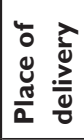 & 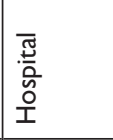 & 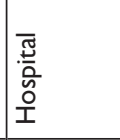 & 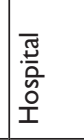 & 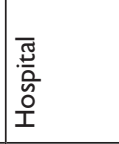 & 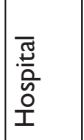 & 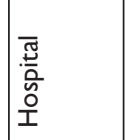 & 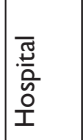 & 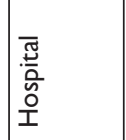 & 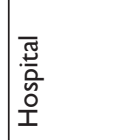 & 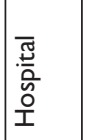 & 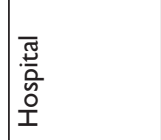 & 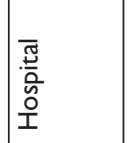 & 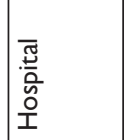 & 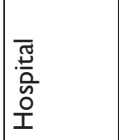 & 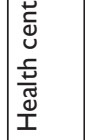 \\
\hline 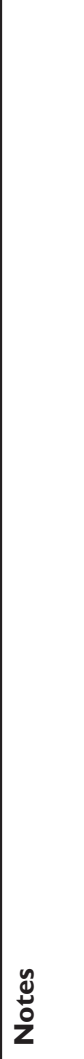 & 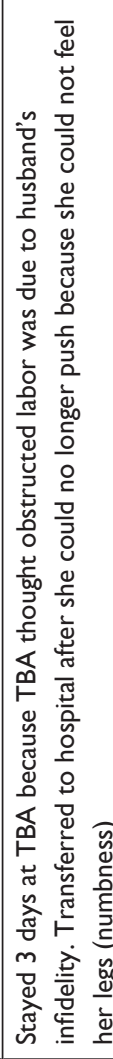 & 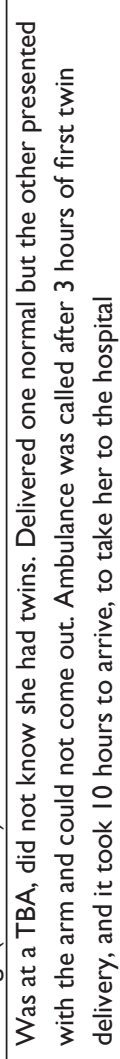 & 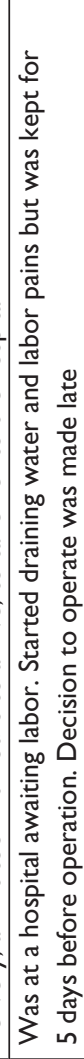 & 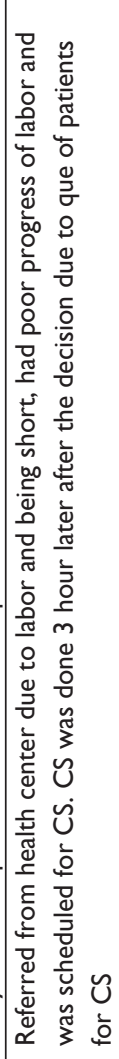 & 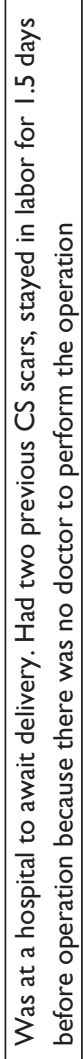 & 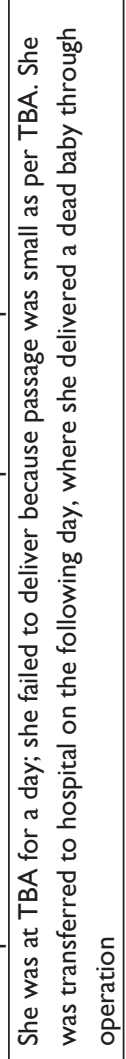 & 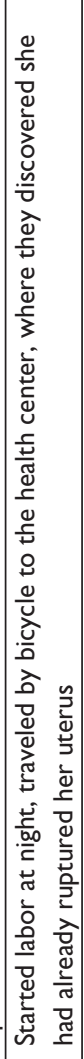 & 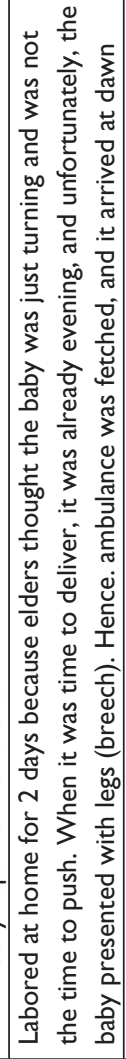 & 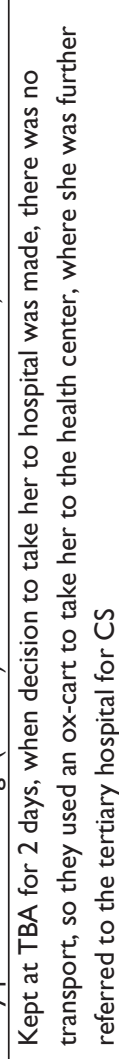 & 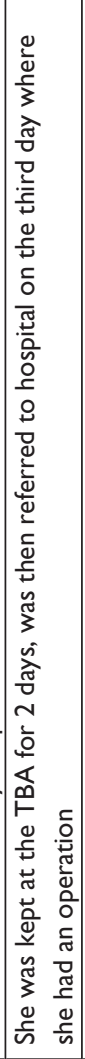 & 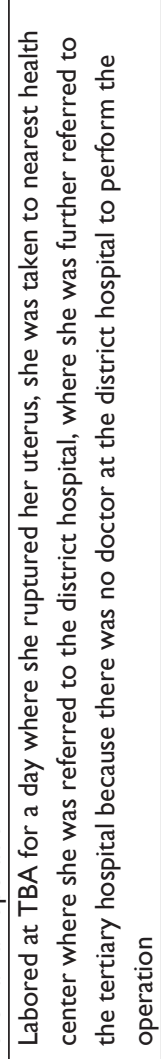 & 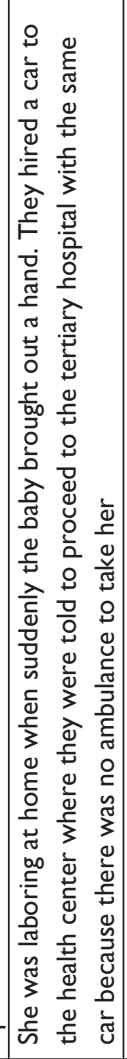 & 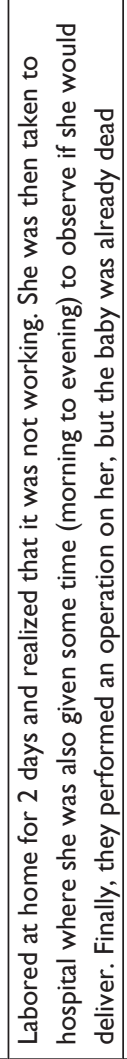 & 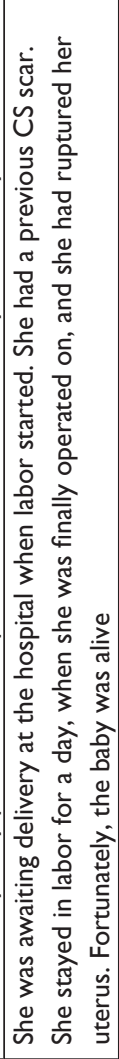 & 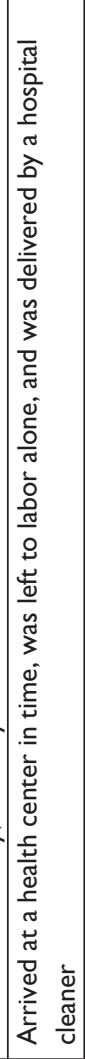 \\
\hline 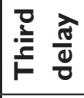 & & & $\times$ & $x$ & $\times$ & & & & & & & & $\times$ & $x$ & $x$ \\
\hline 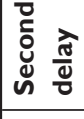 & & $\times$ & & & & $\times$ & $\times$ & $\times$ & $\times$ & & $x$ & $x$ & & & \\
\hline 蒙 & $\times$ & $\times$ & & & & $x$ & $\times$ & $x$ & $\times$ & $\times$ & $\times$ & $x$ & $x$ & & \\
\hline 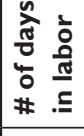 & $m$ & - & in & - & - & N & - & N & N & 0 & - & - & $\mathrm{N}$ & - & - \\
\hline 总 & $\begin{array}{l}\tilde{N} \\
\text { m }\end{array}$ & 吕 & $\begin{array}{l}\tilde{a} \\
\underline{b} \\
\underline{b}\end{array}$ & $\begin{array}{l}\tilde{n} \\
\underline{b} \\
\underline{b}\end{array}$ & $\begin{array}{l}\tilde{y} \\
\underline{b} \\
\underline{-} \\
\end{array}$ & $\begin{array}{l}\tilde{n} \\
\underline{b} \\
\underline{0}\end{array}$ & $\begin{array}{l}\hat{0} \\
1 \\
\mathfrak{y}\end{array}$ & 吕 & $\underset{m}{\tilde{m}}$ & స̃ & $\begin{array}{l}\hat{o} \\
\hat{y} \\
\tilde{y}\end{array}$ & $\begin{array}{l}\hat{p} \\
1 \\
\tilde{y}\end{array}$ & $\begin{array}{l}\hat{0} \\
\hat{y} \\
y\end{array}$ & $\begin{array}{l}\tilde{p} \\
\underline{b} \\
\underline{-}\end{array}$ & \begin{tabular}{|l}
$\tilde{0}$ \\
$\underline{b}$ \\
$\underline{\omega}$
\end{tabular} \\
\hline$\underline{\underline{Q}}$ & - & N & $m$ & t & in & 0 & $r$ & $\infty$ & $a$ & 으 & $=$ & $\simeq$ & $\underline{m}$ & \pm & $\underline{\underline{n}}$ \\
\hline
\end{tabular}




\begin{tabular}{|c|c|c|c|c|c|c|c|c|c|}
\hline$\ddot{~}$ & $\ddot{n}$ & $\ddot{n}$ & $\ddot{\infty}$ & $\ddot{n}$ & $\ddot{n}$ & $\ddot{n}$ & $\ddot{\sim}$ & $\ddot{\sim}$ & $\ddot{n}$ \\
\hline 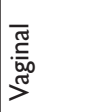 & 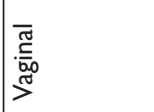 & 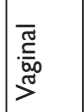 & 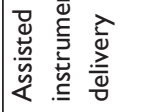 & ○ & 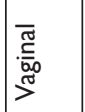 & 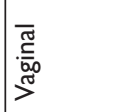 & 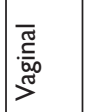 & 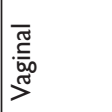 & 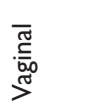 \\
\hline 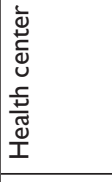 & 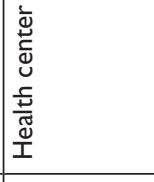 & 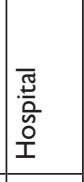 & 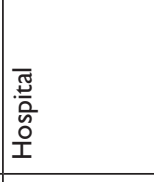 & 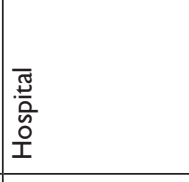 & 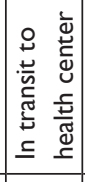 & 造 & 芯 & 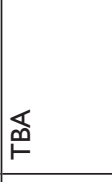 & 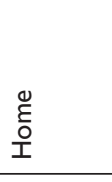 \\
\hline 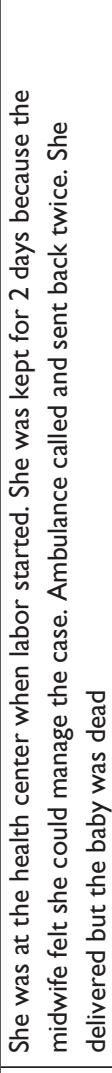 & 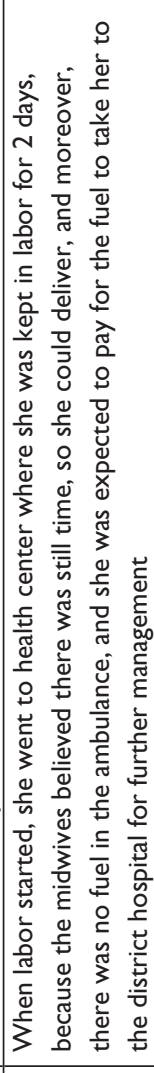 & 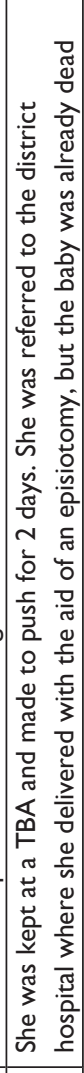 & 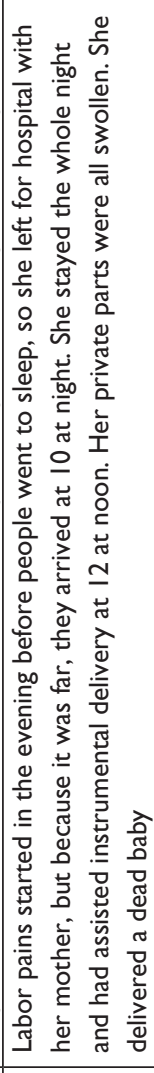 & 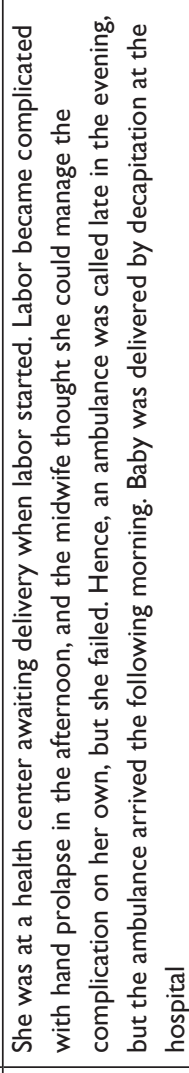 & 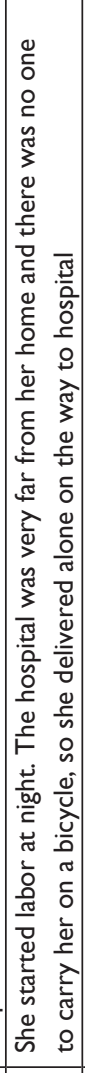 & 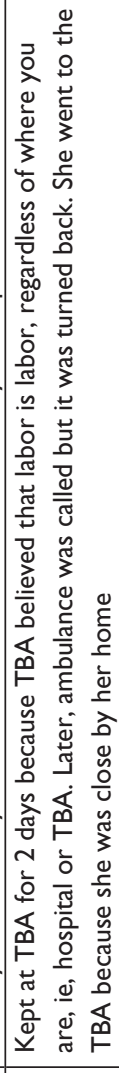 & 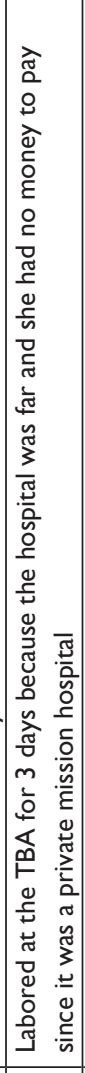 & 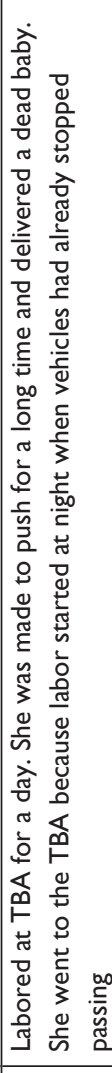 & 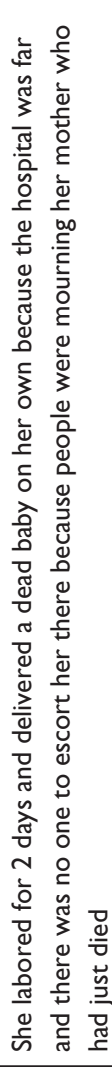 \\
\hline$\times$ & & & $x$ & $\times$ & & & & & \\
\hline & $\times$ & & $\times$ & $\times$ & $\times$ & $\times$ & $\times$ & $\times$ & $\times$ \\
\hline & $\times$ & $\times$ & & & $\times$ & $x$ & $x$ & $\times$ & $\times$ \\
\hline- & $\sim$ & $\sim$ & - & - & - & N & $m$ & - & $\sim$ \\
\hline $\begin{array}{l}\tilde{0} \\
\underline{b} \\
\underline{6}\end{array}$ & $\begin{array}{l}\tilde{w} \\
\underline{b} \\
\underline{-}\end{array}$ & $\begin{array}{l}\tilde{y} \\
\text { m }\end{array}$ & 口 & 弚 & 㒸 & $\underset{m}{\tilde{m}}$ & $\underset{m}{\tilde{m}}$ & $\underset{m}{\tilde{m}}$ & 吕 \\
\hline$\underline{\underline{-}}$ & $\simeq$ & $\stackrel{\infty}{\underline{0}}$ & $\underline{a}$ & 유 & $\bar{N}$ & $\tilde{\approx}$ & $\approx$ & I & $\stackrel{\leftrightarrow}{\sim}$ \\
\hline
\end{tabular}




\section{First delay}

\section{Onset of labor and decision to seek care}

Most of the participants first labored at home before seeking help from elsewhere. Only three participants were already at a health facility awaiting labor when their labor started. The decisions on place of labor and delivery were mostly made by mothers-in-law or sisters-in-law and, in a few cases, by mothers. The choice of place of delivery depended on the time of the day, distance to the health facility, availability of money for transport, money for the service fee in case of private mission hospitals, availability of a bicycle to carry the woman, availability of someone to accompany the woman to the health facility, previous experience with the health facility, and how advanced the labor was. The majority of the participants (10 out of 25) first sought care from the TBAs because they were close enough and/or it was where they received ANC.

One participant summarizing her reasons for choosing to deliver at the TBA said:

To say the truth, right, at the hospital nowadays there are many small children [young midwives] who attend to you, so you think to yourself that, "ah, it is better to go to that old woman to help me." Moreover, at the hospital they are not even there to receive your baby when it is coming, you do the work [push] by yourself and call them when the baby is out. While at the Azamba [TBA], she is always there; she even gives you some herbs to help you deliver fast. And the other thing, the last time I went to deliver at the hospital, I didn't like their high beds, I was always falling when I tried to get on it, and because of that, my baby had difficulty breathing when she was born. [Nasiketi, 15 years living with fistula]

A few participants (three out of 25) labored at home and one of them never sought any help from the hospital, and she delivered alone at home. Recounting her labor experiences one participant said:

My labor and delivery were like this; at that time my mother was sick, and then at night labor pains started and I did not go to the hospital, because people were saying, "who will escort you to the hospital leaving your mother this sick?" Then when she [her sick mother] passed away, they all rushed to the corpse, and nobody stayed to look after me. When they finally came back on the day of my mother's burial, they found the thing [baby] had already fallen out, but already dead. And that was the third day after the onset of the labor pains. [Nambewe, 35 years living with fistula]

\section{Overconfident TBAs as a source of delay}

Until 2007, TBAs were part of the maternal health care delivery system in Malawi. ${ }^{23}$ Women were advised by health care providers to first consult their TBAs when in labor, who would in turn refer them to the hospital in case of a complication. As such, most of the participants in our study reported seeking care from their nearest TBAs. Consequently, due to high volume and increased catchment area, some TBAs became overconfident in their work/skill set. As a result, women with complications were kept for extended periods of time before referral to the hospital, and in individual cases, they were never referred to the hospital at all. Most TBAs failed to recognize or appreciate the severity of the problem at hand, consequently subjecting the women to harmful practices and worse complications.

Summarizing her experience with the TBA, one participant said:

... there [at the TBA], I overstayed without getting better, "a Zamba" [TBA] tried hard, touching me, pressing on the middle of my abdomen [presumably fundal pressure], to help the baby come out, but it did not. So I told her [TBA] to take me to the hospital, but she said, "No, do you think at the hospital they will just pull out the baby without you pushing? Will they not require you to do the same?" Then she [TBA] took a strip of reed and used it to stretch the opening, to widen it, but instead it was just cutting my flesh, and it failed. I was badly wounded. Then they (TBAs) ... they were two of them, said, "Iih, we are cutting her", so they stopped. Then some held my back, some held my mouth and some held my abdomen. Then finally the baby dropped out; but dead. Meanwhile, I was badly injured. (Saying it with such feeling of betrayal). [Nadzimbiri, 12 years living with fistula]

\section{Second delay}

\section{Distance and availability of transportation}

Most participants (15 out of 25) stayed far from a health facility and found it difficult to arrive in time, especially when in labor. Four of these were referred late from the health center due to lack of ambulance and inability of the health care provider to identify the danger signs. Most of our participants lived 10-30 km away from the nearest health facility as observed on home visits during the study. Additionally, place of residence played a great role. Almost half of the participants lived in mountainous areas with no vehicles for transportation, leaving them with a bicycle or an ox-cart as the only option for transport. In some cases, women in labor had to walk to the hospital. One woman living about $8 \mathrm{~km}$ 
from the nearest health center explained how she ended up delivering on the way:

Labor pains started suddenly around midnight, and I started off for the hospital. But since I was all alone and walking slowly struggling along the way due to labor pains, it took time. But if only I had someone, to take me on a bicycle, maybe I would have gotten to the hospital in time. So, while I was on the way to the hospital, my legs got cold; were numb, I could no longer walk. So I thought of just sitting down. Then I just saw that, legs have started coming out. I said “ah!..ah!, what is this?” I tried to stand, but I could not manage. So I just remained seated, all alone. So when the thing [baby] finally stretched its legs and came out, I saw that the thing was already dead. [Nangozo, 13 years living with fistula]

\section{Delayed ambulances}

In cases where the TBA attempted to call for an ambulance, it ended up delaying too. The process of getting an ambulance in itself was time consuming. An individual had to go to the health center by bicycle or on foot to inform the health center staff about the woman at the TBA, who then called the district hospital to send the ambulance to the health center where the messenger would be waiting to give the driver directions to the TBA. One participant explained how her husband went to call for ambulance:

Since we were staying very far from the hospital, the a Zamba [TBA] wrote a letter and gave it to my husband to give it to the doctors at the hospital to send the ambulance. So he went by bicycle and delivered the letter. He came together with the ambulance because he had to direct the driver how to get to the a Zamba. [Nadzimbiri, 12 years living with fistula]

Another participant said:

I arrived at the TBA at 6 o'clock in the evening, the first baby [twin] was born around eight and the second failed to come out. The ambulance was called around 11 o'clock at night, and it arrived around 9 o'clock in the morning; but it was late. [Nasitima, 10 years living with fistula]

\section{Third delay}

Overconfidence of the provider as a source of delay at the health center

Some participants rightfully went to await labor or labor progression and delivery at their nearest health centers, but when a complication occurred, their referrals to the upper health facility level were unnecessarily delayed by the health care providers. Reports from the participants suggested that the health care providers were overconfident and indecisive in the provision of their care. For example, two participants with clear indications for referral were allowed to labor at a health center until it was too late. One participant narrated her experience this way:

When labor started I went to our small hospital [health center]. I stayed there the whole day. They [midwife] called an ambulance, but she then sent it back saying "I will managed her." I stayed and stayed, the ambulance was called again, but the nurse [midwife] sent it back again saying, "I will manage her." [Nanyoni, 4 months living with fistula]

\section{Mismanagement and negligence as predisposing factors to fistula formation}

Mismanagement and negligence at health centers as well as referral hospitals predisposed some women to develop fistula; for example, three participants were separately mismanaged by midwives in different health centers. One was left alone in labor and was delivered by a hospital attendant as she explains in the quote below:

... when we got to the hospital, we found that the doctor [midwife] was not there, she was at her home; the watchman had to go call her. She came and examined me, and told me it was not yet time to push. So she went back home to sleep, meanwhile, the baby was born. The one who helped me was a cleaner, the one who mops the hospital floors, not a real health worker. So she told me that, "the child has injured you". So I told her that, "go and call the doctor [midwife], maybe she can sew the tear right away". And she went, to tell her, and she [midwife] told her [the cleaner] that, "I will see about it tomorrow". That is when she [midwife] sew me. She sew me at around 12:00 noon, but I delivered at night. [Nankhoma, 1 year living with fistula]

Two participants with previous cesarean section scars were in labor for a long period of time, which ultimately led to one of them rupturing her uterus as is reported in the quote below:

My first child I delivered by operation, but the second, third, fourth were normal deliveries. ... the fifth, I rightly went to await labor at the hospital. When I became sick [labor pains], I went to labor and the nurse [midwife] said the passage was not open yet, so we had to wait and observe. 
So while waiting for the passage to open, they did not know that the baby had already caused damage inside. My womb was torn, that was when they operated on me; and told me that "your bladder too has been damaged, so we will send you to Central." [Namajiya, 3 months living with fistula]

\section{Work overload and shortage of staff as a source of delay at the referral health facility}

Heavy workloads and lack of doctors at the referral hospitals increased the amount of time women had to wait to be attended to, further prolonging an already obstructed labor. One participant had to wait for 3 hours for an operation from the time the decision was made. She narrated:

I stayed for three hours after they had prepared me for the operation, because they said there were so many of us waiting for an operation, and I could also see that we were many. [Nambewe, 1 year living with fistula]

Two previous cesarean deliveries are a clear indication for an elective cesarean delivery. ${ }^{29}$ Yet, one participant with two previous cesarean section scars was left to labor for one full day because there was no doctor to operate on her. Expressing her sadness and anger she said:

... I went to wait for labor at the hospital, and the labor pains started while I was already there, but I just cannot understand what the medical people were doing, really. What they did to me was not good, had they done the operation early the child would be alive; even if I had still ended up with this problem. But ah, what was happening, ah ... [Sounding so disappointed]. The doctor who was supposed to do the operation; they said had left; meanwhile I was struggling in great pain, and my body was getting destroyed. They kept on saying, "wait for the doctor; wait for the doctor", without necessarily saying where he went, yet I was in terrible pains.

[Nabengo, 4 months living with fistula]

\section{Delayed referral and condition on arrival to a health facility}

Most participants arrived at the referral hospital in a very critical condition because they had to go through multiple referrals. Participants stated that they had to move from the TBA to the health center and then to the district hospital where sometimes they were further referred to a tertiary level facility for an operation. One participant described her ordeal and her state on arrival to the hospital as follows:

I started laboring at home, at the TBA. So, it happened that things became complicated. The thing (baby) could not be seen here [pointing at her abdomen], my abdomen just became so flat [presumably her uterus had already ruptured] So we started off to our health center. They made me to push, ... then told us that, "No, not here, this needs to go to Dowa [district hospital]". At Dowa, they told us that, "No, here there is no doctor"; so they sent us here at Bottom hospital [tertiary hospital], and did an operation on me; for me to be alive today. ... my lamp had extinguished, when I got here [hospital]. It took me three days to know where I was. [Nabiyeni, 21 years living with fistula]

\section{Consequences of delay: labor outcome, the double loss}

Even though most of the participants eventually got help for their obstructed labor, it was too late for the survival of their babies. Of the 25 participants in the study, only two participants had live babies. A few participants lost their motherhood forever by losing their uteri.

\section{Discussion}

Findings from our study indicate that there was high ANC attendance among our study participants; this is in line with the MDHS report of 2016, which reports ANC attendance of $95 \%{ }^{23}$ However, our findings indicate that when labor started and was complicated, women experienced various barriers to treatment in the event of a complication such as obstructed labor. Most of these barriers were in the form of delays, which is consistent with the findings from previous studies. ${ }^{7,8,15,30}$ In the following sections, we discuss the three delays in the context of existing studies.

\section{The pathway to fistula First delay Onset of labor and decision to seek care}

Consistent with previous studies, when labor started, the women first tried to deliver at home with the help of a relative or a TBA. ${ }^{6} 15$ The women seemed to have limited decisionmaking power as demonstrated by the fact that most of the decisions to seek care were made by their mothers-in-law and, in a few cases, by their mothers. This finding is consistent with the findings from previous studies, ${ }^{15,31}$ but contrary to the findings from a study in Zambia, where the women actively participated in decision making about the place of delivery even though the final decision was made by the husbands. ${ }^{32}$ A possible explanation for this difference could be that with a high level of education as observed among participants in a Zambian study, there will be a significant degree of independency and equality with male partners. Previous research has shown that higher level education and financial autonomy empower women to make household decisions and decisions 
about their own health. ${ }^{33}$ Lack of decision-making power as observed among our study participants could therefore be attributed to their lack of or low levels of education, and poor socioeconomic status, which might have increased their dependency on others for decisions and financial support. On the one hand, as mentioned earlier, in the central region of Malawi, they practice virilocality, ${ }^{21}$ and it might also explain why most decisions were made by the mothers-in-law.

Among all accounts, none of our study participants mentioned their husbands accompanying them or deciding where to deliver their babies when labor started, leading the authors to question their involvement.

In Malawi, like other African countries, pregnancy and childbirth issues have culturally been believed to be "women's issues,"23,34,35 and as such, men tend to be denied some information pertaining to pregnancy and childbirth, ${ }^{35}$ consequently rendering them passive players. Male involvement throughout pregnancy and delivery has been shown to have many benefits including birth preparedness, complication readiness, ability to recognize and respond appropriately to danger signs, increased maternal health utilization, especially skilled birth attendance and postnatal care services, and reduced postpartum depression. ${ }^{36}$ Despite these benefits, husbands of the participants in our study were called for only after labor had been complicated and a decision had already been made by the TBA or the woman's relatives to transfer the woman to the hospital. In such cases, the unprepared husbands had to arrange for an ox-cart or a bicycle or to go to the nearest health center or hospital to request for an ambulance - a process that further prolonged the delay. In the same line, previous researchers ${ }^{29,37}$ have also observed that cultural beliefs, socioeconomic status, distance to the health facility, and previous experiences with the health system may influence decision to utilize maternal health services, which was the case with some participants in our study. In some cases, the choice of the TBAs could be the only option for the women, depending on her condition at the time. ${ }^{7}$

\section{TBAs' contribution to delays}

Malawi has two types of TBAs: trained and untrained. Until 2007, their functions among others included ANC, uncomplicated vaginal deliveries, and referral of women with complications such as prolonged labor. ${ }^{38}$ Unfortunately, nine out of 25 women in our study were kept in labor for unacceptably extended periods of time, leading to injured mothers and dead babies. ${ }^{1}$ This finding is not surprising and is consistent with the findings from previous research. ${ }^{30,39}$ Several factors could explain the delays caused by TBAs in our study. Some TBAs perceived labor as a natural, normal process, not needing urgent care and remedy, thereby missing out on the danger signs. TBAs are trusted and esteemed members of the community; ${ }^{39}$ hence, they would not want to be viewed as incompetent or failures, and consequently lose their respect, which might also explain their behavior. Not surprising, women with complications were rarely referred during the night due to lack of transportation and for security reasons, compounding the delay, and this is consistent with the findings from previous research. ${ }^{30,39}$

Furthermore, we speculate that TBAs could have overlooked their skill limitations ${ }^{30}$ due to overconfidence which comes with years of working experience. Again, TBAs in Malawi tend to be illiterate old women and/or traditional healers, ${ }^{39}$ which in itself could influence decision in one way or the other, especially if untrained.

Additionally, we also speculate that women themselves might have contributed to late referral by deliberately defaulting referral and/or reporting late at the TBA. ${ }^{39}$ However, it was not possible for researchers in our study to visit the TBAs in question to cross-check participants' information or determine what challenges might have contributed to their delayed referrals. This was because the TBAs had died or moved out of the study area at the time of the study.

\section{Second delay}

Except for women who were already at a health facility awaiting labor (7 out of 25), the rest of the women experienced delay in getting to the health facility due to distance and problems with transportation, which was not surprising. The inadequate and poor geographical distribution of health care facilities in the country, poor road infrastructures, and other geographical obstacles such as mountains and rivers might explain this finding. The WHO recommends that for every 500,000 population, there should be five facilities that offer basic emergency obstetric and neonatal care (EmONC) and one facility that offer comprehensive emergency obstetric and neonatal care, ${ }^{40}$ but a recent report on needs assessment on EmONC ${ }^{19}$ indicated that only three districts in the country meet the minimum recommendations. The report ${ }^{19}$ further indicates that only $46 \%$ of the population has access to a formal health facility within a $5 \mathrm{~km}$ radius, and only $20 \%$ of the population lives within $25 \mathrm{~km}$ of a hospital, leaving a greater population with no access to health services, contrary to the universal health access national agenda. ${ }^{41}$ Some participants in our study had to travel $10-30 \mathrm{~km}$ to the nearest health center by bicycle or ox-cart or to a nearby road where they would find a car. Worse still, when they got to the health center, they also had to travel another 50 or more kilometers to the closest facility with the capacity to deal with their case, 
worsening their condition in the process, which is in line with the above report. ${ }^{19}$ Improving the conditions of waiting homes located close to or waiting space within the health facilities might help reduce this type of delay. ${ }^{42}$ In addition, couples and significant others need to be educated on birth preparedness and be encouraged to start saving money for pregnancy and childbirth emergencies to minimize this type of delay.

\section{Third delay}

Findings from our study also suggest that health care providers who by definition are SBAs contributed to fistula formation in one way or another. An SBA is defined as

an accredited health professional such as a midwife, doctor or nurse who has been educated and trained to proficiency in the skills needed to manage normal (uncomplicated) pregnancies, childbirth and the immediate postnatal period, and in identification, management and referral of complications in women and newborns. ${ }^{43}$

The substandard care experienced by women with complications in our study is of great concern, but yet not surprising.

In the EmONC study above, ${ }^{19}$ the researchers found low levels of knowledge and skills among health workers who participated in the study, in all aspects of care of a pregnant woman, which might also help to explain the substandard care observed in our study. However, the finding has a negative implication on health service utilization. If women who seek help from an SBA end up with severe complications such as fistula and stillbirths, they may lose trust in the health care system. ${ }^{29}$ Such loss of trust may consequently affect future utilization of maternal health services, ${ }^{29}$ not only by the woman as a victim but also her social network, thereby undermining the efforts to reduce maternal and neonatal morbidity and mortality. Conversely, knowledge and competency without supportive supervision, equipment, supplies, and functioning ambulances ${ }^{7,8,30,44}$ are unlikely to improve quality of care. However, it was beyond our study to investigate midwives' critical decision-making processes that relate to timely referrals; hence, future research may want to take this into consideration.

Nine of our participants experienced delay when they finally got to a health facility with the capacity to deal with their condition. Since cesarean section is the recommended treatment for obstructed labor, ${ }^{2}$ some women were delayed due to high caseloads for cesarean section and due to shortage of surgeons to perform the cesarean section. This is consistent with the findings from previous studies in Malawi, ${ }^{7,8,44}$ and it reflects the present human resource crisis in the country. Moreover, most of the cesarean sections in district hospitals in Malawi are conducted by a non-physician cadre called COs, who are greatly in short supply; only 640 of the required $2083,{ }^{19}$ which might also explain the shortage. There is a need to strategically plan for training more doctors and COs, who can promptly attend to women with obstructed labor, thereby prevent fistula formation due to delayed treatment.

\section{Consequences of delays on pregnancy outcomes}

The combined effects of the three delays were "near-misses" 45 for some women in our study. Their testimonies of being unaware of their surroundings on arrival to the referral hospital suggest how critical their conditions were. This finding is in-line with the findings from a previous study from Uganda, which found that the majority of the near-misses were from prolonged and obstructed labor. ${ }^{46}$ The WHO defines maternal near miss as "a woman who nearly died but survived a complication that occurred during pregnancy, childbirth or within 42 days of termination of pregnancy." 45 It was beyond the scope of our study to determine how many of the other women actually died from consequences of obstructed labor during the period of our study. However, as already noted in inclusion criteria, all women in our study were survivors of obstructed labor, but all had developed fistula, which left them with leakage of urine and/or feces, smell, and stigmatization, which was likened to death itself. ${ }^{1}$ Worse still, almost all women gave birth to stillborn babies, which is consistent with the findings from previous studies. ${ }^{14,47}$ Unfortunately, women who lose their babies tend to be subjected to emotional stress and social exclusion, ${ }^{11,48}$ instead of emotional support. For instance, in a study to explore values related to neonatal mortality and stillbirths in Ethiopia, the researchers found that women who lost their babies at birth suffered social repercussions, including being blamed, mistreated, and dishonored through divorce. ${ }^{48}$ Similarly, a few participants in our study were blamed for the loss of their babies as it was associated with laziness to push out the baby.

\section{Conclusion and recommendations}

Testimonies by women in our study illustrate the complexity of the journey to fistula formation. Poverty, illiteracy, inaccessible health facilities, negligence, limited male involvement, and shortage of staff together conspire to fistula formation. In order to prevent new cases of OF in Malawi, all the above-mentioned issues need to be addressed. The Birth Prepared and the Complication Readiness (JHPIEGO/MNH Program. Monitoring birth preparedness and complication 
readiness: tools and indicators for maternal and newborn health. In. Baltimore, MD: JHPIEGO; 2004. Available from: https://www.k4health.org/sites/default/files/Monitoring $\% 20$ Birth\%20Prepareness\%20and\%20Complication\%20Readiness\%20toolkit.pdf. Accessed October 7, 2018.) strategy developed by The Johns Hopkins Program for International Education in Gynecology and Obstetrics for the improvement of maternal and neonatal care through the reduction of the three delays has assisted with formulating the following recommendations to address the issues identified in our study.

\section{At an individual level and a family level}

The focus should be to teach women, their spouses, and family members to recognize danger signs, identify a birth location with skilled providers, save money, designate a decision maker, and arrange for transportation. There is need to encourage women in hard to reach rural areas to await labor at their nearest health facilities to avoid delays.

\section{At community level}

There is a need to educate communities on danger signs of pregnancy and childbirth through drama groups as well as community social gatherings to ensure everyone gets acquainted with issues of childbirth regardless of their gender. Especially, male and female members in rural communities should be given lessons on what goes on in a woman's body during pregnancy and mechanisms of childbirth. Such lessons should take into account the community's level of education and their cultural and traditional beliefs and myths concerning pregnancy. This could help both women and men make informed decisions in case of a childbirth complication.

The by-laws that encourage institutional deliveries, which are already operational in most Malawian communities should be encouraged and supported. However, for by-laws to be effective, the health system needs to be very supportive by ensuring that health workers have required equipment, supplies, and functional ambulances and offer standard quality care.

\section{At a health facility level}

There is a need to conduct continuous professional training and workshops, specifically for health care providers in rural areas to update their knowledge and skills, to ensure proper diagnoses and management of maternal complications such as obstructed labor, malposition, and abnormal presentations. This should be supported with regular supervision to ensure that nurse midwives perform and adhere to professional standards.

Finally, larger issues, such as illiteracy and poverty, require a multisector approach to address them. Among other approaches, girl child education needs to be promoted to delay early marriages and increase her bargaining decision-making power in the society. Women's small-scale businesses should be encouraged and supported to increase their financial autonomy and decision making power.

\section{Future research}

Future research might explore midwives' critical decisionmaking processes that relate to timely referrals, as well as knowledge and perceptions about labor and complications among men in rural areas.

\section{Availability of data and materials}

Aggregated data or individual cases will be made available upon request in order to protect our participants looking at how sensitive and stigmatizing fistula is. Meanwhile, some data are already included in the article. Interested parties in this issue should contact the first author (JC).

\section{Acknowledgments}

We are very grateful to the women who participated in the study for their time and information. And special thanks to the staff of BFCC for their support and cooperation during the study. We are grateful to Ekaterina Bogatyreva for editing our manuscript. No fund was received for this study.

\section{Author contributions}

VCT was responsible for the study conception, design, and organization. JC was responsible for data collection, transcription, and supervision of data transcription and translation. All authors contributed to data analysis, drafting and revising the article, gave final approval of the version to be published, and agree to be accountable for all aspects of the work.

\section{Disclosure}

The authors report no conflicts of interest in this work.

\section{References}

1. Wall LL. Dead mothers and injured wives: the social context of maternal morbidity and mortality among the Hausa of northern Nigeria. Stud Fam Plann. 1998;29(4):341-359.

2. Wall L, Arrowsmith S, Briggs N, Lassey A. Urinary incontinence in the developing world: the obstetric fistula. In: Proceedings of the Second International Consultation on Urinary Incontinence, Paris; 2001. Available from: https://www.fistulafoundation.org/pdf/UIDW.pdf. Accessed August 31, 2018

3. Ahmed S, Tunçalp Ö. Burden of obstetric fistula: from measurement to action. Lancet Glob Health. 2015;3(5):e243-e244.

4. WHO [webpage on the Internet]. 10 facts on obstetric fistula. WHO; 2014. Available from: http://www.who.int/features/factfiles/obstetric_fistula/en/. Accessed August 31, 2018. 
5. Roka ZG, Akech M, Wanzala P, Omolo J, Gitta S, Waiswa P. Factors associated with obstetric fistulae occurrence among patients attending selected hospitals in Kenya, 2010: a case control study. BMC Pregnancy Childbirth. 2013;13:56.

6. Bangser M, Mehta M, Singer J, Daly C, Kamugumya C, Mwangomale A. Childbirth experiences of women with obstetric fistula in Tanzania and Uganda and their implications for fistula program development. Int Urogynecol J. 2011;22(1):91-98.

7. Combs Thorsen V, Sundby J, Malata A. Piecing together the maternal death puzzle through narratives: the three delays model revisited. PLoS One. 2012;7(12):e52090.

8. Mgawadere F, Unkels R, Kazembe A, van den Broek N. Factors associated with maternal mortality in Malawi: application of the three delays model. BMC Pregnancy Childbirth. 2017;17(1):219.

9. Kalilani-Phiri LV, Umar E, Lazaro D, Lunguzi J, Chilungo A. Prevalence of obstetric fistula in Malawi. Int J Gynaecol Obstet. 2010;109(3): 204-208.

10. Johnson K. Incontinence in Malawi: analysis of a proxy measure of vaginal fistula in a national survey. Int J Gynaecol Obstet. 2007;99(Suppl 1): S122-S129.

11. Yeakey MP, Chipeta E, Taulo F, Tsui AO. The lived experience of Malawian women with obstetric fistula. Cult Health Sex. 2009;11(5): 499-513.

12. Yeakey MP, Chipeta E, Rijken Y, Taulo F, Tsui AO. Experiences with fistula repair surgery among women and families in Malawi. Glob Public Health. 2011;6(2):153-167.

13. Drew LB, Wilkinson JP, Nundwe W, et al. Long-term outcomes for women after obstetric fistula repair in Lilongwe, Malawi: a qualitative study. BMC Pregnancy Childbirth. 2016;16(1):2.

14. Rijken Y, Chilopora GC. Urogenital and recto-vaginal fistulas in southern Malawi: a report on 407 patients. Int J Gynaecol Obstet. 2007; 99(Suppl 1):S85-S89.

15. Thaddeus S, Maine D. Too far to walk: maternal mortality in context. Soc Sci Med. 1994;38(8):1091-1110.

16. Silverman D. Interpreting Qualitative Data: Methods for Analysing Talk, Text and Interaction. 2nd ed. London: Sage; 2001.

17. Creswell JW. Qualitative Inquiry and Research Design. Choosing among Five Traditions. London: Sage; 1998.

18. World Health Organization [webpage on the Internet]. Malawi; 2017. Available from: http://www.who.int/countries/mwi/en/. Accessed December 5, 2017.

19. USAID, UNFPA, UNICEF, Clinton Health Service Initiative, Save the Children. Malawi Emergency Obstetric and Newborn Care Needs Assessment, 2014; 2015. Available from: https://www.healthynewbornnetwork.org/hnn-content/uploads/Malawi-EmONC-Report-June2015_FINAL.pdf. Accessed September 1, 2018.

20. World Health Organization [webpage on the Internet]. The second ten years of the World Health Organization: 1958-1967; 1968. Available from: http://apps.who.int/iris/handle/10665/39254. Accessed May 26, 2018

21. Berge E, Kambewa D, Munthali A, Wiig H. Lineage and land reforms in Malawi: do matrilineal and patrilineal landholding systems represent a problem for land reforms in Malawi? Land Use Policy. 2014; 41(Suppl C):61-69.

22. Chewa people: the great matriarchal Bantu people of Africa and their ancient Nyau secret society; 2013. Available from: https://kwekudeetripdownmemorylane.blogspot.no/2013/06/chewa-people-great-bantupeople-of.html. Accessed September 1, 2018.

23. National Statistical Office - NSO/Malawi and ICF 2017. Malawi: Demographic Health Survey 2015-2016. Zomba, Malawi: NSO and ICF: National Statistical Office; 2016.

24. Freedom from Fistula. Malawi [webpage on the Internet]. Available from: http://freedomfromfistula.org/projects/malawi. Accessed September 1, 2018.
25. UNFPA, Engender Health. Obstetric Fistula Needs Assessment Report: Findings from Nine African Countries; 2003. Available from: https:// www.engenderhealth.org/wp-content/uploads/imports/files/pubs/ maternal-health/report/fistula-needs-assessment.pdf. Last accessed on 1st September, 2018.

26. Braun V, Clarke V. Using thematic analysis in psychology. Qual Res Psychol. 2006;3(2):77-101.

27. Green J, Thorogood N. Qualitative Methods for Health Research. London: Sage; 2009.

28. World Medical Association [webpage on the Internet]. WMA Declaration of Helsinki: ethical principles for medical research involving human subjects; 1964. Available from: https://www.wma.net/policies-post/ wma-declaration-of-helsinki-ethical-principles-for-medical-researchinvolving-human-subjects/. Accessed October 25, 2017.

29. Lawson JB, Harrison KA, Bergstrom S. Maternity Care in Developing Countries. London: RCOG Press; 2001.

30. Mataya R. Report on the Confidential Enquiry into Maternal Deaths in Malawi (2008-2012). Ministry of Health; 2012. Available from: http://mdsr-action.net/wp-content/uploads/2016/04/MOH_2016_FinalMalawi-CEMD-Report-18-09-14.pdf. Accessed September 1, 2018.

31. Seljeskog L, Sundby J, Chimango J. Factors influencing women's choice of place of delivery in rural Malawi - an explorative study. Afr J Reprod Health. 2006;10(3):66-75.

32. Sialubanje C, Massar K, Hamer DH, Ruiter RA. Reasons for home delivery and use of traditional birth attendants in rural Zambia: a qualitative study. BMC Pregnancy Childbirth. 2015;15:216.

33. Pambè MW, Gnoumou/thiombiano B, Kaboré I. Relationship between women's socioeconomic status and empowerment in Burkina Faso: A focus on participation in decision-making and experience of domestic violence. Afr Pop Stud. 2014;28(2):1146.

34. Lowe M. Social and cultural barriers to husbands' involvement in maternal health in rural Gambia. Pan Afr Med J. 2017;27:255.

35. Kululanga L, Sundby J, Chirwa E, Malata A, Maluwa A. Barriers to husbands' involvement in maternal health care in a rural setting in Malawi: a qualitative study. J Res Nurs Midwifery. 2012;1(1):1-10.

36. Yargawa J, Leonardi-Bee J. Male involvement and maternal health outcomes: systematic review and meta-analysis. J Epidemiol Community Health. 2015;69(6):604-612.

37. Cham M, Sundby J, Vangen S. Maternal mortality in the rural Gambia, a qualitative study on access to emergency obstetric care. Reprod Health. 2005;2(1):3

38. Smit JJ. Traditional birth attendants in Malawi. Curationis. 1994;17(2): 25-28.

39. Bisika T. The effectiveness of the TBA programme in reducing maternal mortality and morbidity in Malawi; 2008. Available from: https://www. ncbi.nlm.nih.gov/pubmed/19024419. Accessed September 1, 2018.

40. Maine D. Monitoring Emergency Obstetric Care: A Handbook. Geneva, Switzerland: World Health Organization; 2009.

41. Government of Malawi. Health Sector Strategic Plan II 2017-2022 Towards Universal Health Coverage. Lilongwe: Ministry of Health; 2017.

42. Sundu S, Mwale OG, Chirwa E. Antenatal mothers' experience of staying in a maternity waiting home at Malamulo Mission Hospital in Thyolo District Malawi: a qualitative, exploratory study. Womens Health Gynecol. 2017;3(1):1-10.

43. WHO. Making Pregnancy Safer the Critical Role of the Skilled Attendant: A Joint Statement by WHO, ICM and FIGO. Geneva: World Health Organization (WHO), Department of Reproductive Health and Research; 2004.

44. Bradley S, Kamwendo F, Chipeta E, Chimwaza W, de Pinho H, Mcauliffe E. Too few staff, too many patients: a qualitative study of the impact on obstetric care providers and on quality of care in Malawi. BMC Pregnancy Childbirth. 2015;15(1):65. 
45. Pattinson R, Say L, Souza JP, van den Broek N, Rooney C [webpage on the Internet]. WHO maternal death and near-miss classifications; 2009. Available from: http://www.who.int/bulletin/volumes/87/10/09071001/en/. Accessed December 6, 2017.

46. Kaye D, Mirembe F, Aziga F, Namulema B. Maternal mortality and associated near-misses among emergency intrapartum obstetric referrals in Mulago Hospital, Kampala, Uganda. East Afr Med J. 2003;80(3): 144-149.

47. Wall LL, Karshima JA, Kirschner C, Arrowsmith SD. The obstetric vesicovaginal fistula: characteristics of 899 patients from Jos, Nigeria. Am J Obstet Gynecol. 2004;190(4):1011-1016.
48. Sisay MM, Yirgu R, Gobezayehu AG, Sibley LM. A qualitative study of attitudes and values surrounding stillbirth and neonatal mortality among grandmothers, mothers, and unmarried girls in rural Amhara and Oromiya regions, Ethiopia: unheard souls in the backyard. JMidwifery Womens Health. 2014;59(Suppl 1):S110-S117.

49. Changole J, Thorsen VC, Kafulafula U. "I am a person but I am not a person": experiences of women living with obstetric fistula in the central region of Malawi. BMC Pregnancy Childbirth. 2017;17(1):433.
International Journal of Women's Health

\section{Publish your work in this journal}

The International Journal of Women's Health is an international, peerreviewed open-access journal publishing original research, reports, editorials, reviews and commentaries on all aspects of women's healthcare including gynecology, obstetrics, and breast cancer. The manuscript management system is completely online and includes

\section{Dovepress}

a very quick and fair peer-review system, which is all easy to use. Visit http://www.dovepress.com/testimonials.php to read real quotes from published authors.

\footnotetext{
Submit your manuscript here: http://www.dovepress.com/international-journal-of-womens-health-journal
} 